\title{
Symptoms and Severity of Visual Stress in Nursing Students: Implications for Education and Healthcare Settings
}

\author{
Stephen J. Loew ${ }^{*}$, Nigel V. Marsh² ${ }^{2}$, Celestino Rodríguez-Pérez ${ }^{3}$, \\ Kenneth Watson' \& Graham L. Jones' \\ 1 University of New England, Armidale, Australia \\ 2 James Cook University, Singapore \\ ${ }^{3}$ University of Oviedo, Spain
}

\begin{abstract}
Visual Stress reportedly affects $5-12 \%$ of the general population and $20-30 \%$ of people with dyslexia. Symptoms are characterized by visual discomfort and perceptual distortions when viewing lines of text, and can be exacerbated by fluorescent lighting and bright paper. In this study, nursing students reported their levels of visual discomfort while reading text on contemporary (ultra-white) paper and on beige-coloured paper, under either standard classroom-lighting (600 lux; $n=31)$ or reduced illumination (400 lux; $n=25$ ). Under the standard lighting, $10(28 \%)$ of subjects reported notable levels of Visual Stress (scoring $\geq 15$ on a visual discomfort scale of 1-30). When reading from the beige-coloured paper the group reported less discomfort across all six symptoms. These differences were statistically significant for five of the six symptoms and the total discomfort score. The results were similar but less pronounced for the group in the reduced illumination condition. This study found that Visual Stress-related reading discomfort can also affect capable readers and, moreover, that simple adjustments to lighting and/or visual media can alleviate such symptoms. The implications of these findings for organisational practice in education and healthcare settings are discussed.

Keywords: Visual stress; Reading difficulties; Fluorescent lighting; Ultra-white paper, Excessive illumination
\end{abstract}

\section{Síntomas y severidad del Estrés Visual en estudiantes de enfermería: implicaciones para los entornos educativos y sanitarios}

Resumen: El estrés visual puede afectar del 5 al 12\% de la población general y del 20 al 30\% de las personas con dislexia. Los síntomas se caracterizan por incomodidad visual y distorsiones de la percepción visual en la lectura, y pueden agravarse con la iluminación fluorescente y el papel brillante. En este estudio, estudiantes de enfermería expresan niveles de incomodidad visual al leer texto en papel ultrablanco y en papel de color beige, ya sea con iluminación estándar del aula (600 lux; $n=31$ ) o con iluminación reducida (400 lux; $n=25$ ). Bajo la iluminación estándar, $10(28 \%)$ de los sujetos informaron niveles notables de estrés visual (puntuación $\geq 15$ en una escala de 1 a 30 ). Al leer en color beige, el grupo presentó menos malestar en los síntomas. Estas diferencias fueron estadísticamente significativas para cinco síntomas y el total de malestar. Los resultados fueron menos pronunciados para el grupo en la condición de iluminación reducida. Encontrándose que la incomodidad de lectura relacionada con estrés visual también puede afectar a lectores expertos y, además, que simples ajustes a la iluminación y/o medios visuales pueden reducirla. Se discuten las implicaciones para la práctica en entornos educativos y sanitarios.

Palabras clave: Estrés visual; Dificultades de lectura; Iluminación fluorescente; Papel ultrablanco, Iluminación excesiva.

Reading involves complex visual and phonological processes; nevertheless, these processes can only begin when the retina

Recibido: 30/12/2020 - Aceptado: 08/01/2021 - Avance online: 18/01/2021 *Correspondencia: Stephen J. Loew.

School of Psychology, University of New England.

Dirección: Armidale, NSW 2351, Australia.

E-mail:sloew@une.edu.au

Loew, S. J., Marsh, N. V., Rodríguez-Pérez, C., Watson, K. \& Jones, G. L. (2021). Symptoms and Severity of Visual Stress in Nursing Students: Implications for Education and Healthcare Settings. Revista de Psicología y Educación, 16(1), 75-87, https://doi.org/10.23923/ rpye2021.01.203 receives photons reflected (or emitted) from the written page. Consequently, while visual comfort when reading can be affected by many external factors, the amount of illumination, the spectral properties of the illumination, and the reflectance properties of the page being read (contrast, brightness) are vitally important. 
In recent decades however, these three fundamental factors influencing visual and reading comfort have changed substantially (Loew, Jones, \& Watson, 2014), particularly regarding the spectral power distribution of interior lighting in general compounded by over-illumination and bright visual media in schools (Winterbottom \& Wilkins, 2009).

With respect to illumination levels, most countries or jurisdictions have specific illuminance guidelines in place, as stipulated by their relevant Standards Authorities (e.g. Australian/New Zealand Standards, British Standards, European Standards). However, not only do these de facto minima notably exceed the levels necessary for good visibility (Cuttle, 2013) and efficient reading (Fotios, 2011), most workplaces and educational facilities have illuminances well above such levels. This may in part be because guidelines stipulating maximum illumination levels appear to be lacking (Boyce \& Wilkins, 2018). Increasing the brightness of room illuminance beyond recommended levels has not been shown to improve reading efficacy; while it has been shown to induce visual discomfort and headaches in susceptible populations (Rea, 1982, 1983; Winterbottom \& Wilkins, 2009).

In the fields of psychology and vision science there has been growing interest in visualsensory anomalies that cannot be explained by known optometric or ophthalmological deficits. One condition that underlies many of these types of visual processing irregularities has been termed variously, Meares-Irlen Syndrome, Pattern-Related Visual Stress, and Scotopic Sensitivity Syndrome; but nowadays the term Visual Stress (VS) is most frequently used (Allen \& Hollis, 2008; Kriss \& Evans, 2005; Loew et al., 2014).

Symptoms of VS often become apparent when susceptible individuals are asked to view high-contrast repetitive striped patterns, and spatial frequencies in the order of 3 cycles per degree of visual field tend to evoke the greatest discomfort (and can also be verified by an increase in visually-evoked signals detected by EEG). In relation to everyday text, the spatial frequencies of the strokes of letters, and between the words and lines of text, all fall within the cycle-frequency range producing maximal visual discomfort for some individuals when viewing square-wave gratings. Within this context, and although reading is an everyday activity, symptoms of VS in susceptible people when reading are typically described as: perceptual distortions of text (e.g., words appear to move, swirl, shimmer, fade or float); rapidly deteriorating reading rate and accuracy; increasing difficulty tracking the lines of text (e.g., reading the wrong line, skipping words); early onset of visual fatigue; headaches and/or migraine; and heightened sensitivity to fluorescent lighting (Hollis \& Allen, 2006; Loew et al., 2014; Robinson, 1994).

On the other hand, the use of spectral filters has been widely reported to alleviate such symptoms (Allen, Evans, \& Wilkins, 2012; Evans \& Allen, 2016; Loew \& Watson, 2012; Noble, Orton, Irlen, \& Robinson, 2004; Singleton \& Henderson, 2007; Wilkins \& Evans, 2009), although this remains controversial (Griffiths, Taylor, Henderson, \& Barrett, 2016; Ritchie, Della Sala, \& Mclntosh, 2011 , 2012). Notably however, studies employing objective measures (such as fMRI imaging) have not only detected hyper-excitability of the visual cortex in subjects with VS, epilepsy, and/or migraine, but have also produced image-based evidence showing marked reductions in this excitation when the same subjects were using optimallyprescribed spectral filters (Chouinard, Zhou, Hrybouski, Kim, \& Cummine, 2012; Huang et al., 2011; Kim, Seo, Ha, \& Kim, 2015; Wilkins, Huang, \& Cao, 2007).

Estimations of the prevalence of VS vary widely, ranging from 5\% (Evans \& Allen, 2016) to as high as $24 \%$ (Jeanes et al., 1997), and several studies have found a 12 $14 \%$ prevalence in unselected samples (Kriss \& Evans, 2005; Loew, Marsh, \& Watson, 2014 ; Loew, Rodríguez, Marsh, Jones, Núñez, \& Watson, 2015). However, diagnosing VS morbidity can be difficult and this may in large part be due to the varying degrees of symptom severity among VS sufferers, which appears to lie on a spectrum best described as a continuum (Evans \& Joseph, 2002). Moreover, VS-related reading difficulties often only become obvious 
to an observer after a subject has continuously read aloud from normal text for 10 minutes or more (Evans \& Allen, 2016; Loew, Marsh et al., 2014; Robinson, 1994); albeit that reading tests designed to detect VS, such as the Wilkins Rate of Reading Test (WRRT), can show an effect in less time (Wilkins, Jeanes, Pumfrey, \& Laskier, 1996).

Misinterpretation of VS morbidity may also potentially occur because similar or identical symptoms have been reported in a number of independent disorders including: developmental dyslexia (Northway, Manahilov, \& Simpson, 2010; Rodriguez-Pérez, GonzálezCastro, Álvarez, Álvarez, \& Fernández-Cueli, 2012; Wright \& Conlon, 2009); attention deficit/hyperactivity disorder (Loew \& Watson, 2013; Taurines et al., 2010); chronic fatigue syndrome (Loew, Marsh et al., 2014; Robinson, McGregor, Roberts, Dunstan, \& Butt, 2001; Wilson, Paterson, \& Hutchinson, 2015) as well as photosensitive epilepsy and migraine (Wilkins, Huang, \& Cao, 2007). In addition, optometric conditions such as accommodation and vergence anomalies or binocular instability can cause similar symptoms (Evans, 2007; Evans \& Allen, 2016). Hence previous research findings suggesting that $12-14 \%$ of the population experience at least moderate levels of VS symptomatology might be viewed with prudence, particularly in light of a recent systematic review (Evans \& Allen, 2016) which noted the diverse diagnostic criteria used in different studies and identified a propensity to over diagnose VS in some cases. To address this issue however, a recent Delphi study (Evans, Allen, \& Wilkins, 2016) has now developed practical diagnostic guidelines based on consensus via a panel of experts.

Moreover, given that visual discomfort (also referred to as asthenopia) appears to be common in university students (Loew et al., 2015; Yekta, Khabazkhoob, Hashemi et al., 2017), and that 'unexplained asthenopia' was found to be quite prevalent $(12.5 \%)$ in 1,448 young schoolchildren examined during The Sydney Myopia Study (Ip et al., 2006), it may well be a latent issue in academic and workplace environments generally. However, VS could also be interpreted as form of asthenopia that includes visual-perceptual distortions when viewing text, though individuals affected by VS are often unaware that the "text instability" they see whilst reading is not experienced by all readers and thus might be unlikely to ascribe their reading difficulties to overhead lighting or the glare from contemporary brilliant white paper (nowadays infused with fluorescent chemicals). Within this context, this study's grounds for hypothesizing that fluorescent lighting and ultra-white paper may cause reading discomfort, visual fatigue and perhaps reading errors in susceptible individuals who are otherwise able readers are briefly reviewed.

\section{FLUORESCENT LIGHTING}

The effects of room illuminance and glare levels upon visual acuity and reading comfort have been an area of research interest for many years (Berman, Jewett, Benson, \& Law, 1997; Berman, Jewett, Fein, Saika, \& Ashford, 1990; Conlon, Lovegrove, Chekaluk, \& Pattison, 1999). Other studies have also investigated whether the irregular spectrum of fluorescent lighting can affect visual acuity tasks, cognition and fatigue (Boyce, 1994; Navvab, 2001, 2002; Wilkins \& Wilkinson, 1991). More recently, some researchers have questioned the appropriateness of the current typically high levels of illumination in workplace and academic settings.

In one such investigation, Winterbottom and Wilkins (2009) measured the illuminance (lux levels) at students' desks in a broad-sample of 90 classrooms spread across 11 schools in the UK. Their study found that the lighting in $88 \%$ of classrooms dramatically exceeded European illuminance recommendations for school classrooms (European Standard EN 124641: 300 Lux). Moreover, $84 \%$ of the classrooms had highly excessive illumination levels $(\geq$ 1,000 lux), at which point visual discomfort can become a significant issue for many individuals (Rea, 1982, 1983). Winterbottom and Wilkins (2009) also emphasised that any negative effects caused by excessive lighting would be inherently compounded by the added glare reflected from bright visual media such as whiteboards. 


\section{ULTRA-WHITE PAPER}

The whiteness of copy paper is important, as paper whiteness provides contrast with the text and can add to the appearance of a document. Logically however, there likely exists a limit to the degree of whiteness required for optimal reading comfort, beyond which further brilliance and contrast might actually cause reading discomfort or visual fatigue for some readers. While book publishers still tend to use either 'cream-white' or 'true-white' shades of paper, the whiteness-levels of today's copy paper now far exceed previously attainable levels (Xerox ${ }^{\mathrm{TM}}$, 2005), and this has occurred due to changes in paper manufacturing practices (Jurič, Karlović, Tomić, \& Novaković, 2013; lodines, 2004) and is unlikely to be related to customer demand (e.g., nobody complains about the inferior 'whiteness' of newspapers).

During the 1990s, a surge in demand for A4 copy paper led to fierce competition among papermakers to secure unprecedented highvolume sales of copy paper to government departments and businesses. However, paper manufacturers had limited scope for marketing their products as 'superior' (i.e., the size or the thickness of A4 copy paper cannot be enhanced); however, the 'whiteness-index' of a brand of paper offers a viable means of distinguishing one brand from the next (lodines, 2004). Consequently, market competition in the paper industry may have led to a spiral of ever increasing brightness (and thus "perceived whiteness") of A4 copy-paper, irrespective of whether any real benefits to reading comfort might be gained. In contrast however, most book publishers maintained their existing levels of paper-whiteness, perhaps because they aim for optimal reading comfort for their customers?

The scale of change that has occurred to reading material in recent years can best be gauged by comparing the past and present technical specifications of paper, as per the most widely utilised whiteness-index internationally: the CIE-Whiteness index 10 100), which was defined by the Commission Internationale de l'Eclairage (CIE) in France.
For a perfect-reflecting non-fluorescent white material, the CIE would be 100, and prior to 1990 the CIE of the whitest papers existing ranged from 75 to 85 (Loew, Jones, et al., 2014). In contrast, current 'ultra-white' papers display CIE measures of $150-170$ on their packaging, which begs the question: 'How can the whiteness of any form of paper exceed the upper limit of 100 on the CIEWhiteness index?' In reality however, this apparent "CIE paradox" only became possible when manufacturers began adding Optical Brightening Agents (OBAs) to modern printing paper. OBAs are fluorescing chemicals designed to capture ultra-violet (UV) light from the non-visible range and re-emit it as additional visible light (primarily blue-light at $457 \mathrm{~nm} \pm 10 \mathrm{~nm}$ (Jurič, Karlović, Tomić, \& Novaković, 2013; Xerox ${ }^{\text {TM }}$, 2005). The key incentive for manufacturers to utilise OBAs is to exploit the fact that the human visual system perceives a slightly higher proportion of blue light as added whiteness (Loew, 2017). Thus, under lighting comprising UV-content (e.g., modern fluorescent lamps; sunlight), a brand of paper with CIE-Whiteness 160can essentially appear (for the reader) to be whiter than the highest rating on the CIE-Whiteness Index (CIE 100); and moreover, this type of paper can actually reflect (and fluoresce) more visible light back to the eyes of the reader than it receives from the light source shining upon it (lodines, 2004; Xerox $^{\text {TM }}$, 2005).

\section{AIMS}

The key goal of the present study was to investigate if, and to what extent, the relatively recent use of brilliant white paper for reading purposes may be compounding the effects of increased levels of illumination due to brighter fluorescent lamps (as well as LEDs). The negative effects of excessive illumination, brightness and glare, and their impact upon visual acuity, visual comfort and reading efficacy have been widely reported (Berman et al., 1997; Berman et al., 1990; Winterbottom \& Wilkins, 2009), as has the prevalence of VS in the community (Evans \& Allen, 2016; Evans \& Joseph, 2002; Loew et al., 2014). 
Furthermore, some researchers have found that bright fluorescent lighting and ultra-white paper can not only cause visual discomfort in proficient readers, but can also increase rates of reading errors recorded in recognised reading tests (Loew et al., 2015). Thus, the present study was designed to estimate mean levels of visual discomfort in undergraduate university students when reading from ultrawhite paper and compare these to levels reported when reading from less-bright (beige) paper. In an effort to separate the effects of fluorescent lighting from those of fluorescent ultra-white paper, the degree of reading discomfort experienced when reading from both paper types was assessed under typical classroom lighting (Study 1: 600 lux), and reduced lighting (Study 2: 400 lux).

\section{METHOD}

The two studies reported here differed with respect to the lighting conditions under which they were conducted. In Study 1 the lighting level (mean illuminance at lecture desks) was $606 \pm 61$ lux, whereas in Study 2 the lighting level was $407 \pm 20$ lux. No participant took part in both studies. In all other aspects the procedures followed in the two studies were the same. Prior to conducting the two main studies, a pilot study was undertaken to test the validity of the questionnaire items in detecting symptoms of visual stress.

\section{PARTICIPANTS}

STUDY 1. The subjects in Study 1 were 37 undergraduate nursing students. Preliminary inspection of the data showed that two subjects gave atypical and extreme responses (e.g., under the white paper condition all their answers were 0 , yet under the non-white paper condition they reported discomfort due to brightness and glare-related factors). Given that the measures were administered in a group condition, it was unclear if they understood the nature of the symptom-level rating method. Hence they were excluded from the analysis. A further four subjects were excluded as they reported having been previously diagnosed with ADHD (two subjects), and previous research has demonstrated symptom overlap between ADHD and VS (Loew \& Watson, 2013), or a non-optometric reading disorder (two subjects). The remaining 31 subjects constitute the sample reported on here. The average age of the Study 1 subjects was 23 years $(S D=8$, range $=17-45$ years). The majority ( $n=29,94 \%)$ were female while the remaining two $(7 \%)$ subjects were male.

STUDY 2. The subjects in Study 2 were 27 undergraduate nursing students. Two subjects were excluded as one reported having been diagnosed with a non-optometric reading disorder, and the other reported having been diagnosed with chronic fatigue syndrome (associated with photophobia). The remaining 25 subjects constitute the sample reported on here. The average age of the Study 2 subjects was 23 years $(S D=8$, range $=18-50$ years). The majority ( $n=24,96 \%$ ) were female and the remaining one $(4 \%)$ subject was male.

\section{MEASURES}

A questionnaire designed to assess symptoms of visual and reading discomfort was completed by the participants while reading from standard white paper (Condition 1), and from beige-coloured paper (Condition 2). The questionnaire comprised a succinct list of six typical VS symptoms of reading discomfort (Table 1) based upon those commonly described by Conlon et al. (1999), Hollis \& Allen (2006), Irlen (1994), Kruk, Sumbler, \& Willows (2008), and Whiting, Robinson, and Parrott (1994). Participants were asked to indicate the degree to which they experienced any of the six listed symptoms while they read a short passage of text under each of the conditions. It is also noteworthy that current symptoms (rather than previous symptoms) have been found to be more reliable predictors of improvements in reading efficacy from the use of spectral filters (Hollis \& Allen, 2006). The symptom-level rating scale utilised in the questionnaire ranged from $0=$ not at all, to 5 = highly noticeable (causes difficulty). 
The questionnaire was designed to be concise, as reading efficiency in individuals with VS is known to deteriorate in a relatively short period of time (Evans \& Allen, 2016; Irlen, 1994; Robinson, 1994), thus a lengthy survey completion time may not be ideal when comparing two reading conditions. However, the short six-symptom questionnaire also provided a seventh comparative measure: a Total Visual Discomfort Score (range $=0$ 30). This added measure was practical given that it has previously been demonstrated (via a two-parameter Rasch rating-scale model producing a uni-dimensional logistic test scale) that visual discomfort can be accurately measured on a single dimension using a discomfort scale of this design (Conlon et al., 1999).

\section{PILOT STUDY}

The responses to the questionnaire from a healthy comparison group $(n=7)$ were compared to those of a group of individuals diagnosed with VS $(n=7)$ by psychologists with recognised accreditations in MearesIrlen syndrome/VS diagnosis. The absence of reading problems, VS symptoms, or any other vision-related deficits in subjects included in the comparison group was verified by way of a pre-trial screening. All participants in the study completed the questionnaire while reading the same sample of text on standard white paper under standard fluorescent lighting.

The Mann-Whitney $U$ tests revealed statistically significant $(p<.05)$ differences on all six symptoms and the total discomfort score for the questionnaire. For all seven differences the median score for the VS group was higher than that for the normal comparison group. This was especially evident for the total discomfort score (range = 0 - 30), with the VS-diagnosed group median score (20.0) being notably higher than that of the comparison group $(6.0 ; p=0.001)$. These results thus provide some evidence for the validity of the questionnaire in detecting the symptoms of VS.

\section{PROCEDURE}

The study sample comprised 64 undergraduate nursing students at a university in south-eastern Australia. All subjects had volunteered to complete a questionnaire while reading samples of text in their regular lecture theatre. Participants were provided with printed information and were fully briefed on the research protocol before signing an informed consent form. The study was approved by the university's Human Research Ethics Committee and was conducted in full accordance with the Code of Ethics of the World Medical Association (Declaration of Helsinki, 2008) for research involving human subjects.

Participants rated levels of reading discomfort under typical lecture-theatre lighting [tri-phosphor fluorescent lamps; correlated colour temperature (CCT): 5,000K; controlling circuitry: $50 \mathrm{~Hz}$ magnetic ballasts (100 Hz flicker-cycle)] while reading text on standard white printing paper [Reflex ${ }^{\text {TM }}$ 'Ultra White'; CIE whiteness (UV inc): 160; fluorescence $\left(\triangle D 65 / 10^{\circ}\right): 25 \%$; opacity: $91 \%$; density: $80 \mathrm{~g} / \mathrm{m}^{2}$ ] and also on coloured printing paper [Reflex ${ }^{\text {TM }}$ 'Sand'; CIE whiteness: N/A (beige colour); fluorescence: $\mathrm{N} / \mathrm{A}$; opacity: $92 \%$; density: $\left.80 \mathrm{~g} / \mathrm{m}^{2}\right]$. The luminance of each paper was measured with a spot photometer (at a distance of $35 \mathrm{~cm}$ ) and the difference in reflected lux-levels was found to be $<2 \%$ (however, photometers are calibrated to have lower sensitivity in the blueviolet bands). The subjects were randomly divided into two similarly sized groups, with each group assigned to complete the visual discomfort questionnaire under one of two illumination levels (600 lux, or 400 lux; window blinds drawn). All other reading conditions remained identical for both groups.

The two samples of text were positioned side by side on each lecture-theatre desk, with leftto-right positioning counter-balanced. This enabled each participant to simultaneously compare two identical reading samples (one printed on standard white paper and the other on beige-coloured paper) while recording 
symptom ratings with respect to both types of paper on a single questionnaire. Each of the two reading sample pages was stapled to a further three blank pages (of identical paper) to exclude possible effects due to desk-surface colour (opacity of reading samples: $>90 \%$ ).

The lecture-theatre walls were primarily white in colour; thus any background-reflected light was essentially neutral with respect to spectral bias. The samples of text utilised were printed in a standard font utilised in most academic settings (Times New Roman; 12-point) and were read from each participant's accustomed reading distance.

\section{DATA ANALYSIS}

The majority of the data from the studies was not normally distributed. Therefore, nonparametric analysis was undertaken. The Mann-Whitney $U$ test was used to analyse the results from the pilot study, and the Wilcoxon signed-rank test was used to analyse the data from the two main studies. All analysis was conducted using two-tailed significance values. Effect size $(r)$ was interpreted using Cohen's (1988) criteria of .1 = small effect, $.3=$ medium effect, and $.5=$ large effect.

\section{RESULTS}

STUDY 1. The results for Study 1 (600 Lux) are presented in Table 1. The median scores in the white paper condition were greater than, or equal to, the scores in the beige paper condition for all six symptoms and the total visual discomfort score. Six of these seven comparisons reached statistical significance. The reduction in symptom severity was statistically significant for symptom one $(p<.0005)$ with a large effect size $(r=.58)$, symptom two $(p=.006)$ with a medium effect size $(r=.35)$, symptom three $(p=.004)$ with a medium effect size $(r$ $=.37)$, symptom five $(p=.021)$ with a small effect size $(r=.29)$, symptom six $(p<.0005)$ with a medium effect size $(r=.48)$, and the total visual discomfort score $(p<.0005)$ with a large effect size $(r=.50)$. The reduction in symptom severity was not statistically significant for symptom four ( $p=.33$ ).

Table 1

Wilcoxon Signed-Rank Test results for a comparison of Reading Discomfort Scores on Standard White and Beige Coloured (Sand) paper under 600 Lux $(n=31)$

\begin{tabular}{|c|c|c|c|}
\hline & \multicolumn{3}{|c|}{ Median score } \\
\hline & White & Beige & Z \\
\hline Symptom 1: Discomfort due to the brightness or glare of the page & 2.0 & 0.0 & $-4.54^{*}$ \\
\hline $\begin{array}{l}\text { Symptom 2: The further you read down the page, the more effort you } \\
\text { require to take in each paragraph }\end{array}$ & 2.0 & 1.0 & $-2.75^{\star}$ \\
\hline $\begin{array}{l}\text { Symptom 3: Print distortions such as fuzziness, blurring, shimmering or } \\
\qquad \text { vibrating of print }\end{array}$ & 1.0 & 0.0 & $-2.88^{*}$ \\
\hline $\begin{array}{l}\text { Symptom 4: The bold-print appears to be slightly raised above the page, as } \\
\qquad \text { though typed with 'thicker' ink }\end{array}$ & 1.0 & 1.0 & -0.97 \\
\hline $\begin{array}{c}\text { Symptom 5: Words appear to be surrounded by a 'white halo', or 'white } \\
\text { blotches' seem to run through the text }\end{array}$ & 0.0 & 0.0 & $-2.30^{*}$ \\
\hline $\begin{array}{l}\text { Symptom 6: Lose your place reading down the page and often start } \\
\text { reading the wrong line, or skip words and must re-read }\end{array}$ & 2.0 & 0.0 & -3.81 * \\
\hline Total visual discomfort score & 9.0 & 3.0 & $-3.92^{*}$ \\
\hline
\end{tabular}


A further implication of these findings relates to the occurrence of VS symptoms in a non-clinical sample of adult university students who are competent readers. The data obtained under the standard lecturetheatre lighting (600 lux) indicated that levels of reading discomfort present in the Study 1 group were considerable, with $13 \%$ of subjects recording total visual discomfort scores $\geq 20$ $(M=21.3$; range $=20-23)$. Such scores suggest clinical levels of VS symptomatology, as they are indistinguishable from those of the pilot study's VS-diagnosed group ( $M=21.7)$. Further, the average total visual discomfort score of these subjects was markedly lower when reading in the beige paper condition ( $M$ $=8.0$; range $=4-11$ ). As all participants in the study were able-reading university students, the above results support the propositions that: (1) Reading efficacy may be impacted by the wide-use of brilliant ultra-white paper, which nowadays has become standard-issue copy paper, and (2) Any resultant symptoms of VS will likely be evident across the broader community.
STUDY 2. The results for Study 2 (400 Lux) are presented in Table 2. The median scores in the white paper condition were greater than, or equal to, the scores in the beige paper condition for all six symptoms and the total visual discomfort score. Three of these seven comparisons reached statistical significance. The reduction in symptom severity was statistically significant for symptom four ( $p$ $=.033)$ with a medium effect size $(r=.30)$, symptom five $(p=.011)$ with a medium effect size $(r=.36)$, and the total visual discomfort score $(p=.031)$ with a medium effect size $(r$ $=.31$ ). Reductions in symptom severity were not statistically significant for symptom one ( $p$ $=.167)$, symptom two $(p=.094)$, symptom three $(p=.072)$, and symptom six $(p=.159)$.

These results also suggest that the amount of illuminance (or brightness) of fluorescent lighting is an important determinant of levels of visual discomfort, as the group showed fewer significant differences on all seven measures (relative to Study 1) when comparing the white and beige conditions under reduced illumination (400 lux). Nonetheless, when

Table 2

Wilcoxon Signed-Rank Test results for a comparison of Reading Discomfort Scores on Standard White and Beige Coloured (Sand) paper under 400 Lux $(n=25)$.

\begin{tabular}{|c|c|c|c|}
\hline & \multicolumn{3}{|c|}{ Median score } \\
\hline & White & Beige & Z \\
\hline Symptom 1: Discomfort due to the brightness or glare of the page & 1.0 & 0.0 & -1.38 \\
\hline $\begin{array}{l}\text { Symptom 2: The further you read down the page, the more effort you } \\
\text { require to take in each paragraph }\end{array}$ & 1.0 & 1.0 & -1.68 \\
\hline $\begin{array}{l}\text { Symptom 3: Print distortions such as fuzziness, blurring, shimmering or } \\
\qquad \text { vibrating of print }\end{array}$ & 1.0 & 1.0 & -1.80 \\
\hline $\begin{array}{l}\text { Symptom 4: The bold-print appears to be slightly raised above the page, as } \\
\qquad \text { though typed with 'thicker' ink }\end{array}$ & 1.0 & 0.0 & $-2.13^{\star}$ \\
\hline $\begin{array}{c}\text { Symptom 5: Words appear to be surrounded by a 'white halo', or 'white } \\
\text { blotches' seem to run through the text }\end{array}$ & 1.0 & 0.0 & $-2.56^{*}$ \\
\hline $\begin{array}{l}\text { Symptom 6: Lose your place reading down the page and often start } \\
\text { reading the wrong line, or skip words and must re-read }\end{array}$ & 1.0 & 1.0 & -1.41 \\
\hline Total visual discomfort score & 6.0 & 2.0 & $-2.16^{\star}$ \\
\hline Note: ${ }^{*} p<.05$ & & & \\
\hline
\end{tabular}


reading in the beige paper condition, the group's discomfort scores revealed statistically significant reductions for two of the six VS symptoms and also in the total discomfort score (Table 2). The incidences of high VS symptomatology in some Study 2 participants were also notable because, despite reduced lighting, in the white paper condition $8.0 \%$ of subjects recorded total visual discomfort scores $\geq 20(M=22.0$; range $=20-24)$. In the beige paper condition however, as also found in Study 1, none of the participants' total visual discomfort scores exceeded levels $\geq 14$.

\section{DISCUSSION}

The context underlying this study is a concern that the contemporary wide use of fluorescent ultra-white paper in schools and workplaces might be impacting reading by adding to an overall trend towards brighter illumination and visual media. The present study measured levels of visual discomfort in competent readers (university students) while reading text on regular ultra-white paper, and compared these to levels of discomfort while reading from a less-bright (beige-coloured) paper. The data obtained in Study 1 revealed that visual discomfort levels differed significantly between the two reading conditions, with greater discomfort reported when reading from ultra-white paper.

In comparison to the white paper condition, when reading from beige-coloured paper under identical illumination (600 lux) the group's scores showed statistically significant decreases in the discomfort levels on five of the six symptoms assessed. The group's total visual discomfort score was also significantly less in the beige paper condition. It was particularly noteworthy that these reductions in visual discomfort were not due to reduced reflected light from the beige paper, as photometric measurements had shown that the two paper types did not differ significantly in luminance levels. It was also of note that the three symptoms most significantly alleviated were also the three symptoms that are perhaps most likely to lead to reading errors, and were: Symptom 1, Discomfort due to the brightness or glare of the page; Symptom 3, Print distortions, such as fuzziness, blurring, shimmering or vibrating of print; and Symptom 6, Lose your place while reading down the page and often start reading the wrong line, or skip words and must re-read.

However, as both fluorescent lighting and over-illumination in general have been reported as being contributing factors in relation to visual discomfort, headaches, migraine (Rea, 1982, 1983; Westland, Pan, \& Lee, 2017), as well as reading difficulties (Loew et al., 2014; Robinson, 1994), Study 2 examined differences between reading from ultra-white paper and beige paper under dimmer illumination levels (400 lux). The purpose of Study 2 was to separate, to some extent, the effects of fluorescent lighting from those of fluorescent ultra-white paper, by decreasing the former while maintaining the latter.

The findings relating to the total visual discomfort scores from both studies suggest a coherent pattern with respect to the two different lighting levels. For example, in the white paper condition, the incidences of total visual discomfort scores indicating high discomfort $(\geq 20)$ and moderate discomfort (10 - 19) showed the following parallel trends in relation to lux levels and visual discomfort, with each being in the expected direction: For 600 lux $13 \%$ of subjects scored $\geq 20$ and a further $36 \%$ scored 10 - 19 (total $=48 \%$ ), and for 400 lux $8 \%$ of subjects scored $\geq 20$ and a further $16 \%$ scored $10-19$ (total $=24 \%$ ).

Similarly, the data obtained from both of the studies showed that the use of beige paper alleviated various symptoms of visual discomfort, yet the degree of benefit appeared to be largely dependent upon the brightness of the illumination. Perhaps this should not be unexpected, as the fluorescing agents in today's white paper require a UV-emitting light source (i.e., fluorescent lighting) in order to re-emit additional visible light by means of fluorescence. Thus, any potential effects of fluorescent ultra-white paper will inherently be amplified by extra fluorescent lamps, and likewise, any effects of fluorescent lighting on reading will likely be amplified if the text is printed on fluorescent white paper. 
Despite the small sample sizes, the results presented here provide further evidence that the trend towards increased (and brighter) fluorescent lighting has become a key ergonomics-related factor that may actually reduce visual and reading comfort, and thus make reading a more arduous task for many individuals. These results further suggest that the less well known fluorescence of modernday printing paper has similar detrimental effects on reading comfort and is likely to compound the negative impact of the relatively recent use of increasingly brighter (i.e., bluer) forms of fluorescent lighting.

Following on from the current results, a longitudinal study of illumination-induced symptoms of visual discomfort in cohorts of undergraduate nurses as they enter the intensely illuminated hospital workplace would be useful. This is particularly so as these future medical professionals will be working in highstress settings where rapid and accurate reading is crucial, and in which long hours, shift-work and fatigue are the norm. Given the welldocumented high incidence of preventable medical errors in hospitals around the world, with many being due to basic communication errors among health professionals (Makary \& Daniel, 2016; Runcima, 2002), this could be a critically important area of investigation. Follow-up studies involving larger participant samples may also be useful and especially if these incorporated objective measures of VS symptomatology such as performance-based tests assessing changes in reading efficiency. These findings could be further substantiated using portable eye-tracking technology, which has recently shown that spectral-filtering can produce large and significant improvements in reading efficacy (Guimareas, Vilhena, Loew, \& Guimareas, 2020). Future research in this area and broader-ranging studies may not only have vital implications for education and healthcare delivery, but may also have the potential to resolve the decades-long contention surrounding VS symptomatology, its diagnosis, and its treatment.

\section{- Conflict of interest.}

The authors declare no conflict of interest

\section{REFERENCES}

Allen, P.M., Evans, B.J.W., \& Wilkins, A.J. (2012). The uses of colour in optometric practice to ameliorate symptoms of visual stress. Optometry in Practice, 13, 1-8.

Allen, P.M., \& Hollis, J. (2008). Meares-Irlen syndrome - a need for increasing awareness in the general public. Ophthalmic and Physiological Optics, 28, 291-292. https:// doi.org/10.1111/opo.2008.28.issue-3

Berman, S.M., Jewett, D.L, Benson, B.R., \& Law, T.M. (1997). Despite different wall colors, vertical scotopic illuminance predicts pupil size. Journal of the Illuminating Engineering Society, 26, 59-68.

Berman, S.M., Jewett, D.J., Fein, G., Saika, G., \& Ashford, F. (1990). Photopic luminance does not always predict perceived room brightness. Lighting Research Technology, 22, 37-41.

Boyce, P.R. (1994). Is full-spectrum lighting special? In J.A. Veitch (Ed.), Full-Spectrum Lighting Effects on Performance, Mood, and Health' (pp. 30-36). IRC Internal Report no. 659, Ottawa: National Research Council of Canada, Institute for Research in Construction.

Boyce, P.R., \& Wilkins, A. (2018). Visual discomfort indoors. Lighting Research \& Technology 50, 98-114.

Chouinard, B.D., Zhou, C.I., Hrybouski, S., Kim, E.S., \& Cummine, J. (2012). A functional neuroimaging case study of Meares-Irlen syndrome/visual stress (MISViS). Brain Topogr, 25(3), 293-307. https://doi.org/10.1007/s10548-011 0212-z

Cohen, J.W. (1988). Statistical power analysis for the behavioural sciences (2nd edition). Hillsdale, NJ: Lawrence Erlbaum Associates. Conlon, E.G., Lovegrove, W.J., Chekaluk, E., \& Pattison, P.E. (1999). Measuring visual discomfort. Visual Cognition, 6, 637-663. https://doi. $\mathrm{org} / 10.1080 / 135062899394885$

Cuttle, C. (2013). A new direction for general lighting practice. Lighting Research and Technology, 45, 22-39.

Declaration of Helsinki [World Medical Association]. (2008). 7th revision of theDeclaration of Helsinki: ethical 
principles for medical research involving human subjects. 59th World Medical Association (WMA) General Assembly, October, 2008, Seoul. http://www. wma.net/en/30publications/10policies/ b3/17c.pdf

Evans, B.J.W. (2007). Pickwell's Binocular Vision Anomalies. Oxford: Elsevier.

Evans, B.J.W., \& Allen, P.M. (2016). A systematic review of controlled trials on visual stress using Intuitive Overlays or the Intuitive Colorimeter. Journal of Optometry, 9, 205-218.

Evans, B.J.W., Allen, P.M., \& Wilkins, A.J. (2017). A Delphi study to develop practical diagnostic guidelines for visual stress (pattern-related visual stress). J Optom, 10(3), 161-168. https://doi. org/10.1016/i.optom.2016.08.002

Evans, B.J.W., \& Joseph, F. (2002). The effect of coloured filters on the rate of reading in an adult student population. Ophthalmic and Physiological Optics, 22, 535-545.

Fotios, S. (2011). Lighting in offices: lamp spectrum and brightness. Coloration Technology, 127, 114 120. https://doi.org/10.1111/i.14784408.201 1.00285.x

Griffiths, P.G., Taylor, R.H., Henderson, L.M., \& Barrett, B.T. (2016). The effect of coloured overlays and lenses on reading: a systematic review of the literature. Ophthalmic \& Physiological Optics, 36, 519-544. https://doi.org/10.1111/ opo. 12316

Hollis, J., \& Allen, P.M. (2006). Screening for Meares-Irlen sensitivity in adults: can assessment methods predict changes in reading speed? Ophthalmic and Physiological Optics, 26, 566-571.

Huang, J., Zong, X., Wilkins, A., Jenkins, B., Bozoki, A., \& Cao, Y. (2011). FMRI evidence that precision ophthalmic tints reduce cortical hyperactivation in migraine. Cephalagia, 31, 925-36.

lonides, G. (2004). Adding value for improved market competitiveness of mechanical printing papers. Pulp \& Paper Canada, 105(4), 95-99.

Ip, J., Huynh, S., Kifley, A., Mitchell, P., Robaei, D., Rose, K., Morgan, I., \& Smith, W. (2006). Impact of parental myopia and near-work on childhood refraction: a comparison of two ethnic groups in the Sydney Myopia Study. 11th International Myopia Conference 2006, United Kingdom: Blackwell Publishers.

Irlen, H. (1994). Scotopic sensitivity/Irlen syndrome: hypothesis and explanation of the syndrome. Journal of Behavioral Optometry, 5, 62-65.

Jeanes, R., Busby, A., Martin, J., Lewis, E., Stevenson, N., Pointon, D., \& Wilkins, A.J. (1997). Prolonged use of coloured overlays for classroom reading. British Journal of Psychology, 88, 531-548.

Jurič, I., Karlović, Tomić, I., \& Novaković, D. (2013). Optical paper properties and their influence on colour reproduction and perceived print quality. Nordic Pulp \& Paper Research Journal, 28(2), 264-273.

Kim, J.H., Seo, H.J., Ha, S.G., \& Kim, S.H. (2015). Functional magnetic resonance imaging findings in Meares-Irlen syndrome: a pilot sudy. Korean J Ophthalmol, 29(2), 121-125. https://doi.org/10.3341/ kjo.2015.29.2.121

Kriss, I., \&Evans, B.J.W. (2005). The relationship between dyslexia and Meares-Irlen syndrome. Journal of Research in Reading, 28, 350-364. https://doi.org/10.1111/ j. 1467-9817.2005.00274.x

Kruk, R., Sumbler, K., \& Willows, D. (2008). Visual processing characteristics of children with Meares-Irlen syndrome. Ophthalmic and Physiological Optics, 28, 35-46. https://doi.org/10.1111/i.14751313.2007.00532.x

Loew, S.J. (2017). Reading conditions in schools: a review of fluorescent lighting, ultra-white paper, unexplained learning difficulties, and visual stress in the classroom. Revista de Psicología y Educación, 12(2), 85-94.

Guimareas, M.R., Vilhena, D., Loew, S.J., \& Guimareas, R.Q. (2020). Spectral overlays for reading difficulties: Improved oculomotor function and reading efficiency among children and adolescents with visual stress. Perceptual and Motor Skills, 127(2), 490-509.

Loew, S.J., Jones, G.L., \& Watson, K. (2014). Meares-Irlen/visual stress syndrome, classroom fluorescent lighting and reading 
difficulties: a review of the literature. Insights on Learning Disabilities, 11, 129169.

Loew, S.J., Marsh, N.V., \& Watson, K. (2014).

The prevalence of symptoms of visual stress/Meares-Irlen syndrome in subjects diagnosed with chronic fatigue syndrome. International Journal of Clinical and Health Psychology, 14, 87-92.

Loew, S.J., Rodriguez, C., Marsh, N.V., Jones, G.L., Núñez, J.C., \& Watson, K. (2015). Levels of visual stress in proficient readers: effects of spectral filtering of fluorescent lighting on reading discomfort. Spanish Journal of Psychology, 18, 1-11.

Loew, S.J., \& Watson, K. (2012). Spectral filtering of fluorescent lighting: effects upon reading and visual discomfort in slow readers. In J. A. Gonzalez-Pienda, C. Rodriguez, D. Alvarez, R. Cerezo, E. Fernandez, M. Cueli, T. Garcia, E. Tuero, \& N. Suarez, (Eds.), Learning Disabilities: Present and Future (pp.267. 278). Massachusetts: Learning Disabilities WorldWide ${ }^{\circledR}$.

Loew, S.J., \& Watson, K. (2013). The prevalence of scotopic sensitivity/MearesIrlen syndrome in subjects diagnosed with ADHD: does misdiagnosis play a role? Croatian Review of Rehabilitation Research, 49, 50-58.

Makary, M.A., \& Daniel, M. (2016). Medical error-the third leading cause of death in the US. British Medical Journal (Online), 353, i2139. https://doi.org/10.1136/ bmi.i2139

Navvab, M. (2001). A comparison of visual performance under high and low color temperature fluorescent lamps. Journal of the Illuminating Engineering Society, 30, 170-175.

Navvab, M. (2002). Visual acuity depends on the color temperature of the surround lighting. Journal of the Illuminating Engineering Society, 31, 70-84.

Noble, J., Orton, M., Irlen, S., \& Robinson, G.L. (2004). A controlled field study of the use of coloured overlays on reading achievement. Aust J Learn Diffic, 9(2), 14-22. https:// doi.org/10.1080/19404150409546760

Northway, N., Manahilov, V., \& Simpson, W.A. (2010). Coloured filters improve exclusion of perceptual noise in visually symptomatic dyslexics. Journal of Research in Reading, 33, 223-230. https://doi.org/hdl.10101/ npre.2008.1729.1

Rea, M.S. (1982). An overview of visual performance. Lighting Design and Application, 12, 35-41.

Rea, M.S. (1983). Effects of Haidinger's brushes on visual performance. Journal of the Illuminating Engineering Society, 12, 197-203.

Ritchie, S.J., Della Sala, S., \& Mclntosh, R.D. (2011). Irlen colored overlays do not alleviate reading difficulties. Pediatrics, 128, e932-e938. https://doi. org/10.1542/peds.2011-0314

Ritchie, S.J., Della Sala, S., \& Mclntosh, R.D. (2012). Irlen Colored Filters in the Classroom: A 1-Year Follow-Up. Mind, Brain, and Education, 6(2), 7480. https://doi.org/10.1111/i.1751228X.2012.01139.x

Robinson, G.L. (1994). Coloured lenses and reading: a review of research into reading achievement, reading strategies and causal mechanisms. Australian Journal of Special Education, 18, 3-14.

Robinson, G.L., McGregor, N.R., Roberts, T.K., Dunstan, R.H., \& Butt, H. (2001). A biochemical analysis of people with chronic fatigue who have Irlen syndrome: Speculation concerning immune system dysfunction. Perceptual and Motor Skills, 93, 486-504.

Rodriguez-Pérez, C., González-Castro, P., Álvarez, L., Álvarez, D., \& Fernández-Cueli, M. (2012). Neuropsychological analysis of the difficulties in dyslexia through sensory fusion. International Journal of Clinical and Health Psychology, 12, 69-80.

Runciman, B. (2002). Lessons from Australia. In S. Emslie, K. Knox, \& M. Pickstone (Eds.). Improving Patient Safety: Insights from American, Australian and British Healthcare (pp 47-53). ECRI, Welwyn.

Singleton, C., \& Henderson, L.-M. (2007). Computerized screening for visual stress in children with dyslexia. Dyslexia, 13(2), 130-151. https://doi.org/10.1002/ dys.329

Taurines, R., Schmitt, J., Renner, T., Conner, A.C., Warnke, A., \& Romanos, M. (2010). 
Developmental comorbidity in attentiondeficit/hyperactivity disorder. ADHDAttention Deficit Hyperactivity Disorder, 2, 267-289. https://doi.org/10.1007/ s1 2402-010-0040-0

Westland, S., Pan, Q., \& Lee, S. (2017). A review of the effects of colour and light on non-image function in humans. Coloration Technology 133, 349-361.

Whiting, P.R., Robinson, G.L.W., \& Parrott, C.F. (1994). Irlen coloured filters for reading: a six year follow-up. Australian Journal of Remedial Education, 26, 13-19.

Wilkins, A.J., \& Evans, B.J.W. (2009). Visual stress, its treatment with spectral filters, and its relationship to visually induced motion sickness. Applied Ergonomics, 41, 509-515. https://doi.org/10.1016/i. apergo.2009.01.011

Wilkins, A.J., Huang, J., \& Cao, Y. (2007). Prevention of visual stress and migraine with precision spectral filters. Drug Development Research, 68, 469. 475. https://doi.org/10.1111/i.15264610.2006.00585.x

Wilkins, A.J., Jeanes, R. J., Pumfrey, P.D., \& Laskier, M. (1996). Rate of Reading Test ${ }^{\circledR}$ : its reliability, and its validity in the assessment of the effects of coloured overlays. Ophthalmic and Physiological Optics, 16, 491-497.
Wilkins, A.J., \& Wilkinson, P. (1991). A tint to reduce eye-strain from fluorescent lighting? Preliminary observations. Ophthalmic and Physiological Optics, 11, 172-175.

Wilson, R.L., Paterson, K.B., \& Hutchinson, C.V. (2015). Increased vulnerability to pattern-related visual stress in myalgic encephalomyelitis. Perception, 44, 14221426.

Winterbottom, M., \& Wilkins, A.J. (2009). Lighting and discomfort in the classroom. Journal of Environmental Psychology, 29, 63-75. https://doi.org/10.1016/i. jenvp.2008.11.007

Wright, C.M., \& Conlon, E.G. (2009). Auditory and visual processing in children with dyslexia. Developmental Neuropsychology, 34, 330-355. https://doi. org/10.1080/875656409002801882

Xerox Corporation. (2005). Demystifying three key paper properties: Whiteness, Brightness, and Shade. Xerox Corporation: Webster, New York 14580, USA.

Yekta, A., Khabazkhoob, M., Hashemi, H., Ali, S., Ostadimoghaddam, H., Najafi, A., \& Heravian, J. (2017). The prevalence of asthenopia and its determinants in a population of university students. Acta Ophthalmol, $95 . \quad$ https://doi. org/10.1111/i.1755-3768.2017.0F008 